\title{
Solar rotational modulations of spectral irradiance and correlations with the variability of total solar irradiance
}

\author{
Jae N. Lee ${ }^{1,2, *}$, Robert F. Cahalan ${ }^{2}$, and Dong L. $\mathrm{Wu}^{2}$ \\ 1 Joint Center for Earth Systems Technology, University of Maryland, Baltimore County, Baltimore, MD, USA \\ 2 NASA Goddard Space Flight Center, Greenbelt, MD, USA \\ ${ }^{*}$ Corresponding author: jae.n.lee@nasa.gov
}

Received 29 January 2016 / Accepted 15 August 2016

\begin{abstract}
Aims: We characterize the solar rotational modulations of spectral solar irradiance (SSI) and compare them with the corresponding changes of total solar irradiance (TSI). Solar rotational modulations of TSI and SSI at wavelengths between 120 and $1600 \mathrm{~nm}$ are identified over one hundred Carrington rotational cycles during 2003-2013.

Methods: The SORCE (Solar Radiation and Climate Experiment) and TIMED (Thermosphere Ionosphere Mesosphere Energetics and Dynamics)/SEE (Solar EUV Experiment) measured and SATIRE-S modeled solar irradiances are analyzed using the EEMD (Ensemble Empirical Mode Decomposition) method to determine the phase and amplitude of 27-day solar rotational variation in TSI and SSI.

Results: The mode decomposition clearly identifies 27-day solar rotational variations in SSI between 120 and $1600 \mathrm{~nm}$, and there is a robust wavelength dependence in the phase of the rotational mode relative to that of TSI. The rotational modes of visible (VIS) and near infrared (NIR) are in phase with the mode of TSI, but the phase of the rotational mode of ultraviolet (UV) exhibits differences from that of TSI. While it is questionable that the VIS to NIR portion of the solar spectrum has yet been observed with sufficient accuracy and precision to determine the 11-year solar cycle variations, the temporal variations over one hundred cycles of 27-day solar rotation, independent of the two solar cycles in which they are embedded, show distinct solar rotational modulations at each wavelength.
\end{abstract}

Key words. Solar rotational modulation - Total solar irradiance - Spectral solar irradiance - SORCE - SATIRE-S

\section{Introduction}

Although solar variability in many different timescales is documented (Lean 1991, 1997; Fröhlich \& Lean 2004; Solanki et al. 2005; Unruh et al. 2008; Domingo et al. 2009; Krivova et al. 2011; Lean \& DeLand 2012; Wehrli et al. 2013; Woods et al. 2015; and references therein), the exact magnitude and the spectral dependence of variations relative to that of total solar irradiance (TSI) are still in debate. In particular, significant differences in SSI variability between observations and models (Ermolli et al. 2013) indicate that there is still uncertainty in our knowledge of how the SSI varies over different timescales and wavelengths.

TSI is the solar energy flux density outside Earth's atmosphere at a distance from the Sun of 1 Astronomical Unit $(\mathrm{AU})$, given in SI units of Watts per square meter $\left(\mathrm{W} \mathrm{m}^{-2}\right)$. The absorption of the TSI, the total energy input to the Earth, determines the Earth's radiation budget and mean temperature (Stephens et al. 2012; Wild et al. 2012). Where this radiative energy is deposited into the different layers of the climate system will be determined by how the incoming solar radiation is distributed with wavelength, and how much variation occurs at each wavelength.

The contributions of ultraviolet (UV) to TSI are relatively small, i.e., less than 10\% (Krivova et al. 2006), but the UV portions of the solar irradiance are quite variable and extremely important to Earth's middle atmosphere through photochemical processes (Haigh 2007; Gray et al. 2010; Haigh et al. 2010;
Merkel et al. 2011; Swartz et al. 2012; Ermolli et al. 2013; Wen et al. 2013; Li \& Tung 2014; and references therein). Solar cycle driven modulations of Arctic and North Atlantic Oscillations (AO/NAO) by the state of the stratosphere have been addressed in many previous works (Shindell et al. 2001; Kodera 2002; Lee \& Hameed 2007; Lee et al. 2008; and references therein). Recent modeling studies suggest that especially the variability of UV is closely linked to sea level pressure changes associated with $\mathrm{AO} / \mathrm{NAO}$ (Kren et al. 2014; Andrews et al. 2015; Ineson et al. 2015). For the near infrared (NIR) region, spectral composition of the solar irradiance provides top of the atmosphere (TOA) constraints on the direct input for atmospheric heating rates. The variations in solar spectral output have the potential to affect the atmospheric temperature structure through complex and non-linear chemical and dynamical processes.

The understanding of the SSI variability is significantly important for climate modeling, but also hindered by the lack of consistent long-term measurements. The variability of SSI and its connection with TSI were explored with satellite measurements and models (Deland \& Cebula 2012; Lean \& Deland 2012; Wehrli et al. 2013; Ball et al. 2011, 2014; and references therein). Some models (e.g. Lean et al. 2005; Krivova \& Solanki 2008) suggest inverse solar cycle changes in IR irradiance, i.e., anti-correlation between TSI and IR. The UV and VIS irradiance variances are suggested to be in-phase (positively correlated) with TSI (Ermolli et al. 2013). While the long-term behavior of solar spectrum can 
only be derived through careful tracing of one instrument to the other based on good knowledge of the degradation of the individual radiometers operating in space, the current observation from space is adequate enough to monitor short-term variability.

The main drivers of solar variability are thought to be magnetic features at the solar surface (Cook et al. 1980; Spruit 1994). Sunspots, forming the hearts of active regions, clearly are dark (Solanki 2003; Rempel \& Schlichenmaier 2011) while the small magnetic elements that form the faculae in active regions and the network elsewhere on the Sun are bright, particularly near the limb and at wavelengths formed above the solar surface (Lagg et al. 2010). It was shown that the variations in Fraunhofer lines define the amplitude of the solar brightness variability, while contributions from continuum were relatively weak, on timescales greater than a day (Unruh et al. 1999; Shapiro et al. 2015). It was suggested that antiphase relationship between SSI variation and solar cycle can be caused by a negative correlation of the continuum intensity with magnetic field observed at some wavelengths (Topka et al. 1997) or by a change of the temperature gradient in the photosphere (Harder et al. 2009; Fontenla et al. 2011).

Since 2003 to present, observations from SORCE (Solar Radiation and Climate Experiment)/TIM (Total Irradiance Monitor), SIM (Spectral Irradiance Monitor), SOLSTICE (Solar-Stellar Irradiance Comparison Experiment), and TIMED (Thermosphere Ionosphere Mesosphere Energetics and Dynamics)/SEE (Solar EUV Experiment) provide the total and spectral solar irradiance measurements with unprecedented accuracy and spectral coverage to determine the amount of solar irradiance reaching at the top of the atmosphere and how solar irradiance is distributed in different wavelengths. While continued calibration and validation is needed in order to improve the accuracy and precision to determine low frequency of solar variations, i.e., the 11-year solar cycle, the amplitudes and phases of 27-day solar rotational variation can be determined in a relatively short period compared to the 11-year solar cycle. These values are less sensitive to instrument degradation uncertainties. The SORCE and TIMED measurements allow cycle-by-cycle comparisons of the solar rotational variation between TSI and SSI for more than one hundred cycles.

In this study, we characterize the SSI rotational variation from SOLSTICE, SIM, SEE observations, and from the SATIRE-S model by comparing the amplitudes and phases of these solar rotational variations in different wavelengths from $120 \mathrm{~nm}$ to $1600 \mathrm{~nm}$. To determine and compare the TSI and SSI variation on rotational timescales, we apply Ensemble Empirical Mode Decomposition (EEMD; Huang et al. 1998) and extract the temporal variation of total and spectral irradiance.

\section{Data}

\subsection{TSI record}

\subsubsection{SORCE/TIM}

The TIM instrument onboard SORCE, launched in January 2003, is an electrical substitution active cavity radiometer, designed to produce measurements with a relatively high accuracy $(350 \mathrm{ppm}$ or $0.035 \%)$ and stability (less than $10 \mathrm{ppm} / \mathrm{yr}$ or $0.001 \% / \mathrm{yr}$ ) (Lawrence et al. 2000). The active cavity is exposed to the Sun, and a voltage applied to equalize its temperature to that of a reference cavity kept at constant temperature. TIM is the first TSI instrument to enable a "phase sensitive detection" operational mode that involves periodic solar exposure of the active cavity by opening and closing its shutter at a fixed frequency, then detecting the response at that shutter frequency, with a shutter frequency chosen to minimize background noise, typically about $1 \mathrm{~min}$. The TIM has four cavities, with one undergoing daily solar exposure, and others exposed more rarely, with a schedule designed to accurately determine the dependence of degradation on exposure (Kopp \& Lawrence 2005; Kopp \& Lean 2011; Kopp et al. 2007, 2012).

\subsection{SSI record}

\subsubsection{SORCE/SOLSTICE}

The SORCE SOLSTICE is a follow-on to the SOLSTICE on the Upper Atmospheric Research Satellite (UARS; Rottman 1999; Floyd et al. 2003) and it measures the daily solar spectral irradiance in UV from 115 to $320 \mathrm{~nm}$ with a resolution of $0.1 \mathrm{~nm}$, an absolute accuracy of better than $5 \%$, and a relative accuracy of $0.5 \%$ per year (McClintock et al. 2005a, 2005b; Snow et al. 2005). The SOLSTICE measurements are made using a pair of identical spectrometers, SOLSTICE A and SOLSTICE B. Each spectrometer independently makes ultraviolet spectral measurements in two intervals: far ultraviolet (FUV) in 115-180 nm and mid-ultraviolet (MUV) in $180-320 \mathrm{~nm}$. The absolute calibration of this instrument relies on the observation of reference stars.

\subsubsection{SORCE/SIM}

The SIM measures the solar irradiance as a function of wavelength continuously over a range covering nearly $97 \%$ of the TSI (Harder et al. 2009). SIM is the only instrument providing continuous observations in the IR with sufficient precision to determine true solar variations. Spectral measurement of solar radiation from SIM shows that solar irradiance changes over 11-year cycle are wavelength dependent. The SIM measurements suggest that solar cycle variations at some VIS and NIR wavelengths, in contrast to TSI, are out of phase with solar activity.

\subsubsection{TIMED/SEE}

The SEE instrument is on the TIMED mission. SEE measures solar spectral irradiance from 0.1-194 nm (Woods et al. 2005) since 2002 to present. We analyze Lyman- $\alpha$ line measured by EUV Grating Spectrograph (EGS) channel of the SEE, which covers wavelengths from 27 to $194 \mathrm{~nm}$ with a spectral resolution of $0.4 \mathrm{~nm}$. The data used in this study are SEE version 11, level 3 (http://lasp.colorado.edu/lisird/ssi/).

\subsubsection{SATIRE-S}

SATIRE-S (Spectral And Total Irradiance REconstruction for the Satellite era) is the most detailed of the SATIRE family of models (Krivova et al. 2011), which rely on the availability of magnetograms and continuum intensity images. Essentially, the solar irradiance is calculated by integrating over the solar surface, accounting for the presence of dark (sunspots) and bright (faculae and network) surface magnetic features. The SSI is reconstructed with three instruments: Kitt Peak Solar Observatory (KP) 512-channel magnetograph and 
spectromagnetograph (SPM), and Michelson Doppler Imager (MDI) which is onboard the Solar and Heliospheric Observatory $(\mathrm{SoHO})$ spacecraft.

The SSI reconstruction covers wavelengths between 115 and $160,000 \mathrm{~nm}$ from 1974 to 2013. Independent from observed solar irradiance, SATIRE-S provides consistent and reliable model-based SSI by identifying four solar surface components from the solar surface magnetic features: the background quiet sun, the dark penumbral and umbral components of sunspots, and faculae. Details on the description of the model/data used here are described in Ball et al. (2014) and Yeo et al. (2014).

\section{Method: ensemble empirical mode decomposition (EEMD)}

The EEMD analysis is applied to the daily mean TSI and SSI time series obtained from TIM, SEE, SOLSTICE, SIM, and SATIRE-S. For the comparison, the TSI and SSI values for missing days from each instrument are interpolated by linear regression. Empirical mode decomposition (EMD) is an algorithm which decomposes data into a finite set of functions called intrinsic mode functions (IMFs) and a trend. It identifies all extremes of a signal and connects upper/lower extremes to form an upper/lower envelope to calculate the spline functions. By subtracting the local mean of the envelope from the signal and iterating this process on the residual, each mode can be identified until the signal satisfies the following conditions of an IMF: (1) In the whole data set, the number of extrema and the number of zero crossings must either equal or differ at most by one. (2) At any data point, the mean value of the envelope defined using the local maxima and the envelope defined using the local minima is zero. A complete description on this method can be found in Huang et al. (1998).

While traditional Fourier analysis decomposes data into fixed amplitudes and frequencies (Eq. (1)), EMD can decompose time series as a sum of the orthogonal empirical modes that have time-variable amplitudes $\left(A_{j}(t)\right)$ and time-varying frequency $\left(\omega_{j}(t)\right)$, by using the Hilbert-Huang transform (HHT) in Eq. (2). This method is more effective than traditional Fourier analysis to extract the frequency of the rotational variation, because solar rotation is not a rigid body rotation but a differential rotation with varying frequency.

$$
\begin{gathered}
X(t)=\sum_{j} A_{j} \exp \left(i w_{j} t\right), \\
X(t)=\sum_{j} A_{j}(t) \exp \left(i \int w_{j}(t) \mathrm{d} t\right) .
\end{gathered}
$$

These periodic modes obtained by EMD do not need to be sinusoidal so as to eliminate the artificial sub-harmonic components due to non-sinusoidal periodical variations. Each mode has a symmetric envelope defined by the local maxima and minima so that its mean amplitude is zero throughout the time domain. Its mean period can be determined by counting the number of local maxima and local minima. Each mode is equivalent to an adaptively filtered signal in an empirically determined frequency range.

To avoid potential overlaps of frequency modes extracted from the data, we average an ensemble of 1000 EMD realizations obtained from the data plus white noise. The white noise contribution to the extracted modes is expected to average down substantially with the 1000-member ensemble till the last mode is identified. The noise analysis allows us to identify the significant modes in the data, even in cases where sharp changes occur in the frequency and time domain. Also, it can help to order the modes with respect to their frequency. The added white noise would populate the whole timefrequency space uniformly with the constituting components of different scales separated by the modes. This noise-assisted EMD analysis method is called ensemble empirical mode decomposition (EEMD). A complete description of EMD can be found in $\mathrm{Wu} \&$ Huang (2004).

This analysis has been successful in extracting the nonlinear, non-stationary 27-day rotational variation in UV irradiance (Ruzmaikin et al. 2007), and in TSI (Lee et al. 2015). This method has been applied in many different fields for spectral analysis in medical engineering (e.g., Liang et al. 2005) and solar cycle analysis (Barnhart \& Eichinger 2011). Detailed description on this method and significance test with TSI data can be found in Lee et al. (2015).

The evolution of the modes from the EEMD analysis of SOLSTICE measured Lyman- $\alpha$ line $(121 \mathrm{~nm})$ is shown, as an example, in Figure 1. The SSI at this wavelength can be fully represented by the IMFs obtained by nine EEMD modes. The last mode characterizes a non-linear trend in the signal. This trend can be used as an estimate of the 11-year solar cycle variation.

Figure 2 shows the probability density functions (PDFs) of instantaneous frequencies of the Lyman- $\alpha$ line decomposition. Mode 4 has a maximum distribution of frequency, $f$, close to 0.034 (1/day), which corresponds to the 29-day period $(f=1 / 29$ day). Similarly, modes 3 and 5 have peaks at 0.054 and 0.015 , which represent 18-day and 66.6-day period, respectively. The temporal variation of $\sim 29$-day mode accounts for $\sim 6 \%$ of the total SOLSTICE Lyman- $\alpha$ line variations over its mission period.

A significance test is performed by applying the EMD analysis on data sets with purely white noise. Comparisons of spectral mode powers from the real data and white noise data determine the statistical significance of each mode extracted. When the white noise significance test is applied, as suggested by Wu \& Huang (2004), all the modes near 13.5-, 27-, and 54-day periods are statistically significant within the $95 \%$ level.

\section{Discussions and results}

During the SORCE mission period, TIM, SIM, and SOLSTICE have continuously monitored the decline of total solar irradiance in solar cycle (SC) 23 , through the solar cycle minimum and solar maximum of SC24, and are currently measuring the declining phase of SC24. Both total and spectral solar irradiance data can be separated into high frequency variation with shorter than 500-day periods, and low frequency variation with longer than 500-day periods. To separate high and low frequency variations in spectral irradiance, we applied the EEMD analysis to the TSI and SSI data, as described in Section 3.

Figure 3 shows a comparison of the high and low frequency variations in TIM measured TSI and SOLSTICE measured Lyman- $\alpha$ at $121 \mathrm{~nm}$. For low frequency variations in the TSI and Lyman- $\alpha$, only modes with longer than 500 -day of periods (i.e., mode 8-9 and trend in Fig. 1) are 

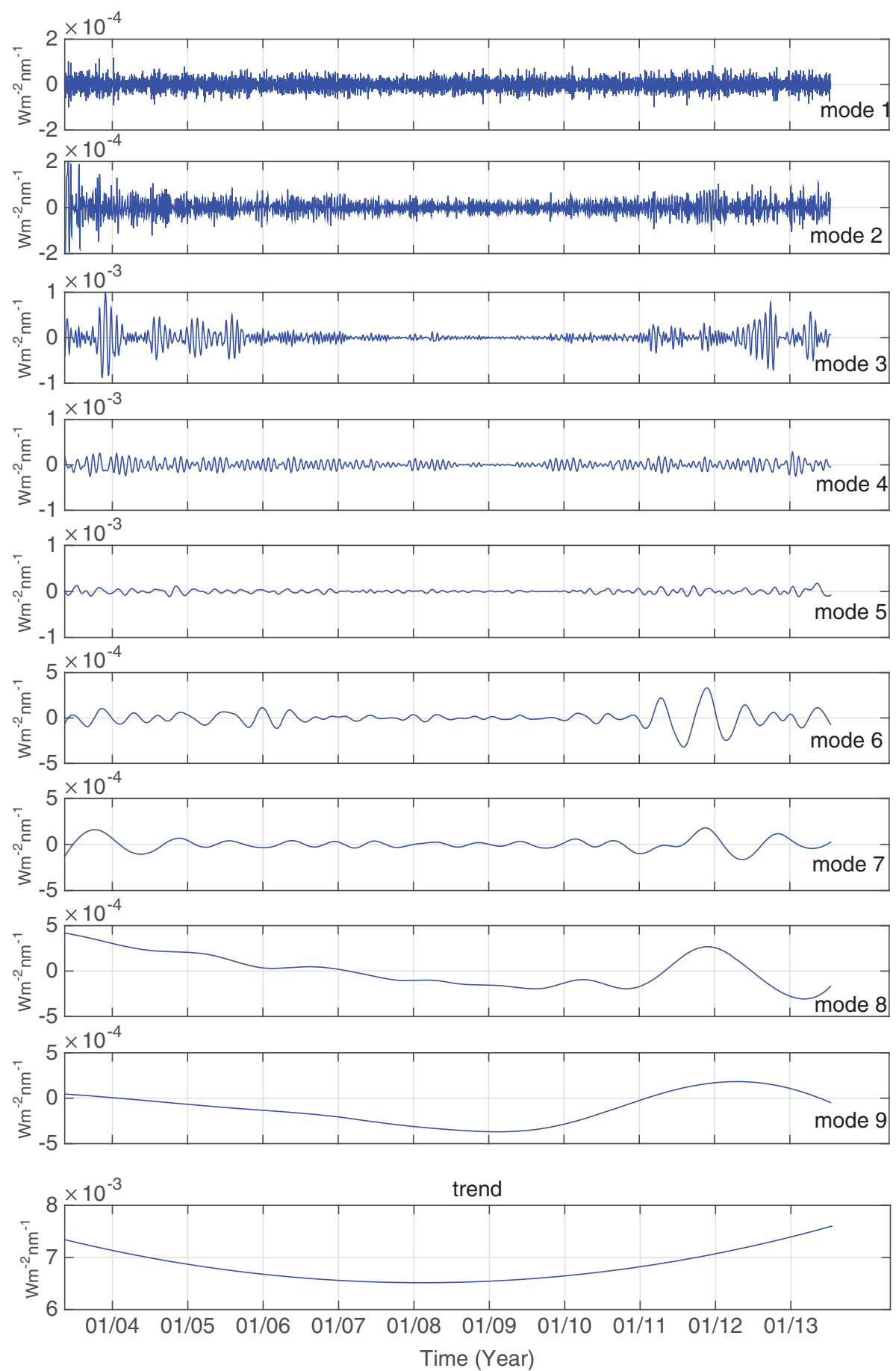

Fig. 1. The time evolution of the EEMD modes, from mode 1 to mode 9 of the SOLSTICE Lyman- $\alpha$ line. Noise-like modes 1 and 2 are also plotted.

considered for each data record. To estimate the low frequency variations, the high frequency modes with shorter than 500-day of periods are removed from the daily averaged data records. Also shown in Figure $3 a$ as dotted lines are low frequency modes with longer than 200-day of periods (i.e., mode 7-9 and trend in Fig. 1). The comparison of the modes between TSI and Lyman- $\alpha$ shows two features. Low frequency variations of TSI and Lyman- $\alpha$ are in phase with the 11-year solar cycle (Fig. 3a), while the high frequency variation between the two is not always in phase with each other (Fig. 3b).
The relative amplitudes of the low frequency variations of TSI and Lyman- $\alpha$ are in good agreement during the current analysis period, as shown in Figure 3a. Both were at high levels early in the SORCE mission, decreased to low values in 2008-2009, and have rebounded at the maximum of SC 24 in 2013 , to the higher values observed early in the mission in 2003-2004. The high values in 2003-2004 are measured during the declining phase of SC23 but not at the maximum of SC 23, since the actual peak of SC 23 was earlier in 2000 and preceded the launch of SORCE. The absolute minimum of TSI in this analysis is $1360.6 \mathrm{~W} \mathrm{~m}^{-2}$ at the end of 2009 , 
J.N. Lee et al.: Solar rotational modulations of spectral irradiance

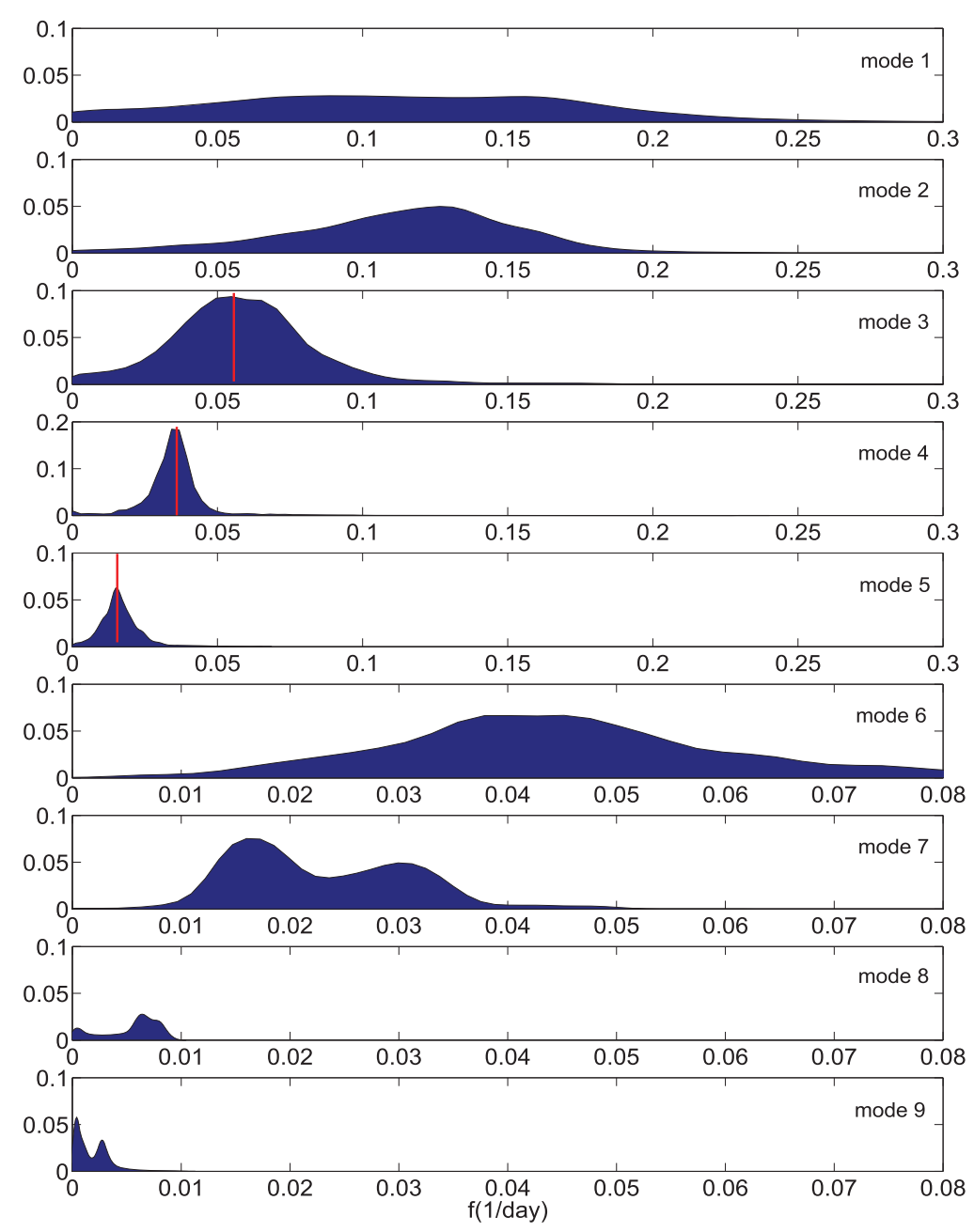

Fig. 2. The probability density function (pdf) of the instantaneous frequencies of each mode of SOLSTICE/Lyman- $\alpha$ (121 nm) line. The histogram is smoothed with a KS (kernel smoothing) density function. The vertical red lines in modes 3-5 mark the harmonics of the solar rotation period, which correspond to $\sim 13.5, \sim 27$, and $\sim 54$ days. Maximum frequency distribution occurs at the following frequencies; 0.054 , 0.034 , and 0.015 in mode 3, 4, and 5, representing 18-day, 29.2-day, and 66.6-day period, respectively.

similar to the value of $1360.8 \pm 0.5 \mathrm{~W} \mathrm{~m}^{-2}$, which is shown by Kopp \& Lean (2011). The absolute minimum value of Lyman- $\alpha$ is $6 \times 10^{-3} \mathrm{~W} \mathrm{~m}^{-2} \mathrm{~nm}^{-1}$ at the middle of 2009 , about a few months earlier than that of the TSI minimum.

In addition to variations with longer than 500-day periods, the low frequency irradiance variations with longer than 200-day periods are also shown in Figure 3a. The 200-day threshold is selected because a solar active region typically takes 5-7 months to decay and disperse back into the quietSun network (Preminger \& Walton 2005; Woods et al. 2015). During high solar activity periods, i.e., 2003-2005 and 2011-2013, TSI modulations with longer than 200-day periods are noticeable. If the TSI variation is the consequence of multiple region outbursts, this modulation of longer than 200 days indicates that a large and strong solar active region at near solar maximum can be active more than 7 months ( 200-day) before decaying. The Lyman- $\alpha$ variations also show similar modulations during high solar activity time, but their amplitudes are less than those of TSI. The 500-day mode of Lyman- $\alpha$ after 2012 is not lower than that of 200-day. This fact indicates that the signs of instrument degradation contributions are not found during the current analysis periods, at Lyman- $\alpha$ wavelength. During low solar activity periods at near solar minimum of SC23 (2007-2009), both TSI and
Lyman- $\alpha$ show variations with small amplitudes. Different from high solar activity periods, the modulation amplitudes of Lyman- $\alpha$ are roughly equal or slightly more than those of TSI.

Comparison of the high frequency variations with shorter than 500-day periods is also shown in Figure $3 \mathrm{~b}$. The maximum magnitude of the TSI high frequency variation $\left(\sim 5 \mathrm{~W} \mathrm{~m}^{-2}\right)$ is roughly seven times the magnitude of the low frequency variation $\left(\sim 0.7 \mathrm{~W} \mathrm{~m}^{-2}\right)$ during the SORCE period. The magnitude of the high frequency variation of Lyman- $\alpha\left(\sim 2.5 \times 10^{-3} \mathrm{~W} \mathrm{~m}^{-2}\right)$ is roughly equal to or slightly more than the magnitude of the low frequency variation $\left(\sim 1.7 \times 10^{-3} \mathrm{~W} \mathrm{~m}^{-2}\right)$ during the same period. The magnitudes of high frequency variations are smaller during low solar activity periods than high solar activity periods when many more and larger active regions exist on the solar surface. The amplitudes of high frequency modulations are also minimum during solar minimum years (2008-2009) when there are very little active regions existing on the solar surface. This result is consistent with the previous findings (Fioletov 2009; Dikty et al. 2010) that the 27-day solar forcing amplitude is weaker during solar minimum.

The solar spectrum originates at different levels of the solar atmosphere. The VIS ( $\sim 500 \mathrm{~nm})$ radiation originates low in the 
(a)

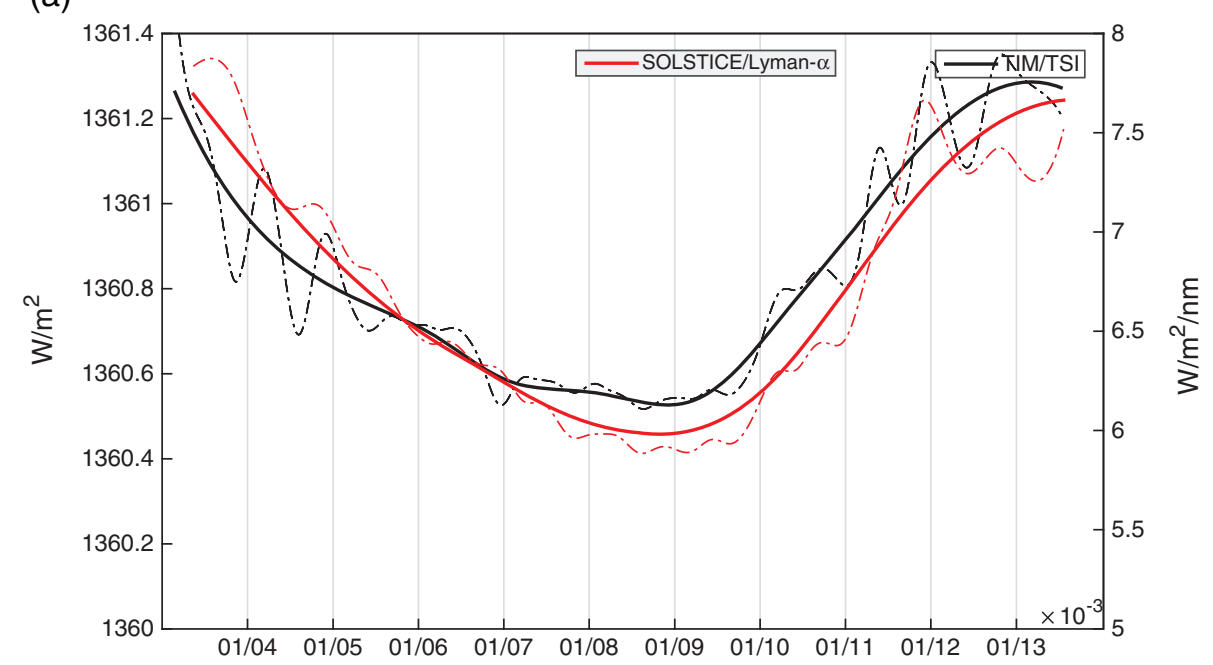

(b)

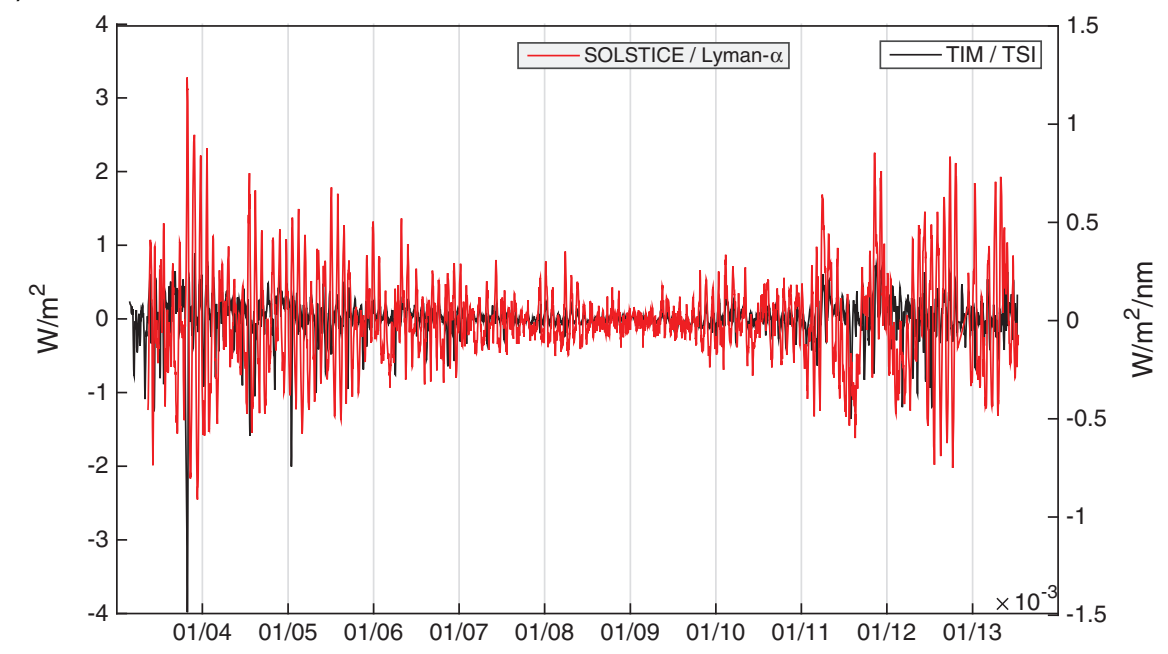

Fig. 3. Comparison of (a) low frequency variation with longer than 500-day of periods from daily averaged TIM/TSI and SOLSTICE/Lyman- $\alpha$ $(121 \mathrm{~nm})$ in black and red lines. Overlaid dotted lines are low frequency variations with longer than 200-day of periods, for TSI and Lyman- $\alpha$, respectively. Numbers in left and right axis indicate variations in $\mathrm{W} \mathrm{m}^{-2}$ for TSI and $\mathrm{W} \mathrm{m}^{-2} \mathrm{~nm}^{-1}$ for Lyman- $\alpha$, respectively. (b) Same as (a), but high frequency variation which indicates the short-term variation. For this mode, the low frequency variations longer than 500 -day of periods are removed from TSI and Lyman- $\alpha$ time series.

photosphere, near $200 \mathrm{~nm}$ it originates near the top of the photosphere, and near $160 \mathrm{~nm}$ it originates slightly above the photosphere where the temperature of the solar atmosphere has decreased to its minimum value (Rottman et al. 2001). The shorter wavelengths of UV, e.g., Lyman- $\alpha$ are emitted from the higher layers of the chromosphere and transition region. These emission lines arise in higher temperature layers of the upper photosphere and chromosphere and are strongly related to the magnetic activity of the Sun in plage regions (Lean 1991). The short wavelengths of the spectrum like Lyman- $\alpha$ contribute only a very small fraction of TSI (less than $10^{-5}$ of TSI). However, they originate in the more active and disturbed levels of the higher solar atmosphere; they can vary by quite large factors compared to their fraction in TSI. The absolute amplitude of Lyman- $\alpha$ low frequency variation is only $\sim 1.7 \times 10^{-3} \mathrm{~W} \mathrm{~m}^{-2} \mathrm{~nm}^{-1}$, nearly 400 times smaller than that of TSI. Detailed analysis of rotational variations in TIM/TSI and its comparison with those from ACRIM/ACRIM III and SOHO/VIRGO TSI are presented in Lee et al. (2015).

To further study the amplitude and phase of short-term variation in UV and their relationship to those of TSI, the relative amplitudes of the 27-day variations obtained by EEMD method are shown in Figures 4, 5, and 7. Figures 4 and 5 display standardized rotational amplitudes of TSI and UV for a single selected wavelength, at Lyman- $\alpha$ and $240 \mathrm{~nm}$, respectively. The 27-day solar rotation in TSI is obtained from SORCE/TIM, and those in UV are obtained from SOLSTICE $A$ and SEE at Lyman- $\alpha$, and SOLSTICE B and SIM at $240 \mathrm{~nm}$. The wavelength of $240 \mathrm{~nm}$ is chosen since SIM has overlapping observations with SOLSTICE $\mathrm{B}$ in that wavelength. The SOLSTICE provides measurements from 112 to $320 \mathrm{~nm}$ with $1 \mathrm{~nm}$ resolution, and SIM provides measurements from 240 to $2400 \mathrm{~nm}$ with $\sim 0.1 \mathrm{~nm}$ to $\sim 11 \mathrm{~nm}$ resolution.

The standardized amplitudes are estimated with an intrinsic mode function $I$ for mode 4 in Figure 1, by removing the mean of $I$ over the analysis time and by dividing with its standard deviation,

$$
A_{\mathrm{TSI}}(t)=\frac{I_{\mathrm{TSI}}(t)-\overline{I_{\mathrm{TSI}}}}{\sigma\left(I_{\mathrm{TSI}}\right)}, \quad A_{\mathrm{UV}}(t)=\frac{I_{\mathrm{UV}}(t)-\overline{I_{\mathrm{UV}}}}{\sigma\left(I_{\mathrm{UV}}\right)} .
$$



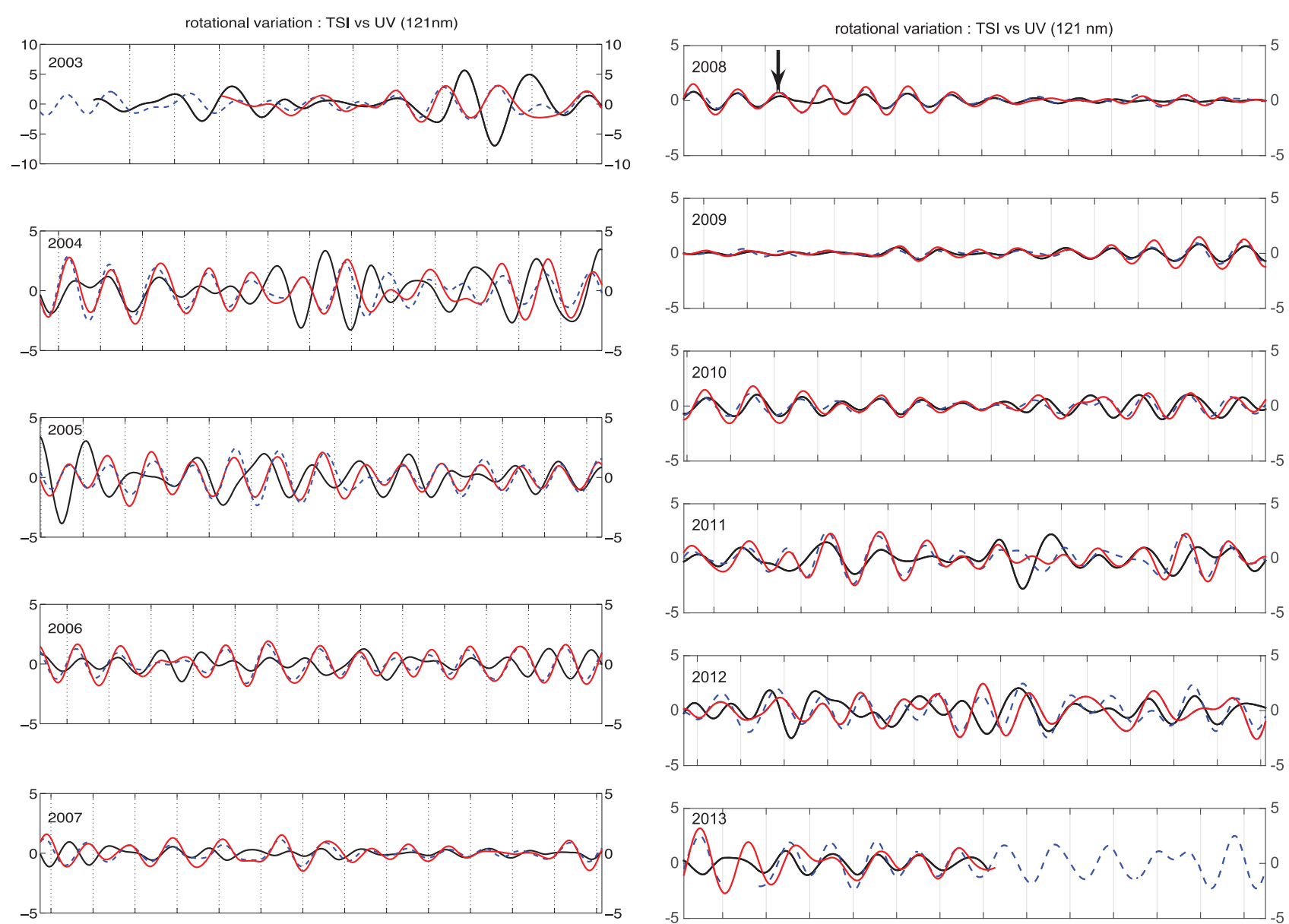

Fig. 4. Comparison of rotational variation from the TIM TSI (black), SOLSTICE Lyman- $\alpha$ (red), and TIMED/SEE Lyman- $\alpha$ (blue dot) at $121 \mathrm{~nm}$ during 2003-2013. Vertical lines denote Carrington rotational cycle. Black arrow in early 2008 indicates the time of 2008 outburst, when a single active region is dominating the irradiance variation. The normalized amplitudes are estimated with an intrinsic mode function $I$, by removing the mean of $I$ over the analysis time and by dividing with its standard deviation, for TSI and Lyman- $\alpha$ line, respectively. Numbers in right and left axes indicate the normalized amplitude of the rotational variations of TSI and Lyman- $\alpha$.

As shown in Figures 4 and 5, the phase of the rotational mode of UV exhibits differences from that of TSI, especially during high solar activity periods. For example, many of the UV and TSI modes were out of phase during 2003 and 2005 and during 2011 and 2013. At near solar minimum during late 2007 and 2010, they were mostly in phase with each other even the amplitudes of the modes were relatively small. The out-ofphase relationship between UV and TSI rotational variations can be explained by the timescale of solar active region influence on TSI. A solar active region typically appears suddenly and then takes 5-7 months to decay and disperse back into the quiet-Sun network (Preminger \& Walton 2005). TSI and UV can be out of phase when new active regions emerge when the sunspots are larger and darker.

When a single active region is dominating the irradiance variation (e.g., early 2008), the Lyman- $\alpha$ and UV emissions shorter than $250 \mathrm{~nm}$ exhibit strong 27-day rotation peaks when the active region first appears and then the rotational peaks decrease with each rotation until the active region impact is hard to detect after five solar rotations (Woods et al. 2015). For the TSI, there is a strong decrease of irradiance when the sunspot is near disk center. After 1-2 months, the sunspots have dispersed mostly into the active network and thus appear as bright photospheric features. Then for the subsequent solar rotations, the TSI variation indicates only bright contributions and is in phase with the Lyman- $\alpha$ and UV variations. This feature in the 2008 outburst is clearly shown in Figure 4, as marked with a black arrow. The TSI mode (in black curve) disappears in third Carrington cycle in 2008, then it is in phase again with the Lyman- $\alpha$ mode in subsequent rotational cycles. The TSI mode does not show negative phase for this cycle, where marked as a black arrow, since short-term variability of less than 27-day frequency is removed by EEMD. Because many active regions can be on the Sun at the same time during high solar activity periods, the rotational modes of TSI and UV can be out of phase when the sun is active. This feature is also shown in the MUV at $240 \mathrm{~nm}$ in Figure 5.

The rotational modes estimated from two independent UV measurements show very similar features. The comparison of the 27-day rotation of UV at Lyman- $\alpha$ estimated from SOLSTICE A and SEE shows good agreement both in amplitude and phase (Fig. 4). While the SOLSTICE mode deviates in the first couple of weeks, it generally agrees with the SEE measurements for the remainder of the time. Similarly, the comparison of the 27-day rotation of UV at $240 \mathrm{~nm}$ estimated from SOLSTICE B and SIM is in good agreement after the 

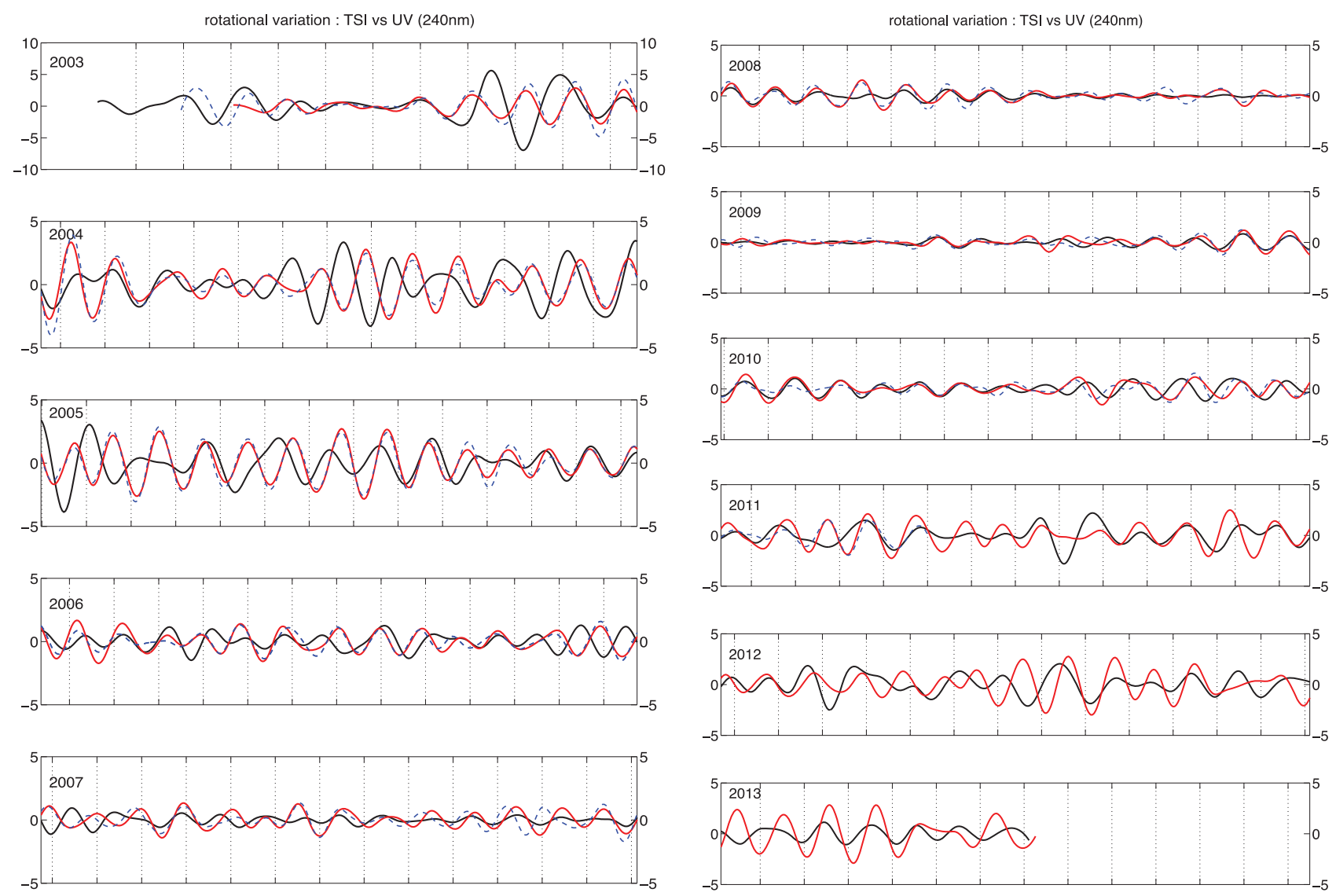

Fig. 5. Same as Figure 4, but comparison of rotational variation from the TIM TSI (black), SOLSTICE UV (red), and SIM UV (blue dot) at $240 \mathrm{~nm}$ during 2003-2013. The normalized amplitudes are estimated with an intrinsic mode function $I$, by removing the mean of $I$ over the analysis time and by dividing with its standard deviation, for TSI and UV, respectively. Numbers in right and left axes indicate the normalized amplitude of the rotational variations of TSI and UV.

first few weeks of deviation, both in amplitude and phase, as shown in Figure 5.

The solar rotational modulation is a manifestation of the distribution of activity on the solar disk, related to the appearance and disappearance of active regions on the solar disk (Richard et al.; poster presented at SORCE meeting ${ }^{1}$ ), not the global mean activity of the Sun. Changes in the Sun's radiative output are primarily related to bright, irregular patches seen on the Sun's surface. In general, these active regions are the direct result of internal magnetic disturbances that erupt through the visible photosphere of the Sun, and the characters of these active regions change with height of the solar atmosphere. Extended regions of brightening are referred to as faculae as seen in the photosphere (VIS and NIR). These features exhibit only small brightness enhancement when seen on the disk of the Sun, but greater enhancement near the limb. Within the active regions the much smaller sunspots have even more intense magnetic field strength and lower brightness compared to emission from the neighboring photosphere. At photospheric wavelengths $(256 \mathrm{~nm}<\lambda<500 \mathrm{~nm})$ a competition thus exists between the enhanced emission from faculae and reduced emission from the sunspots. In this case, the TSI may be low while UV is high with the intensified magnetic field. A possible explanation is that this contrast may lead the off-phase of

\footnotetext{
${ }^{1} \mathrm{http} / / /$ lasp.colorado.edu/sorce/news/2008ScienceMeeting/posters/ P1_08_Richard.pdf
}

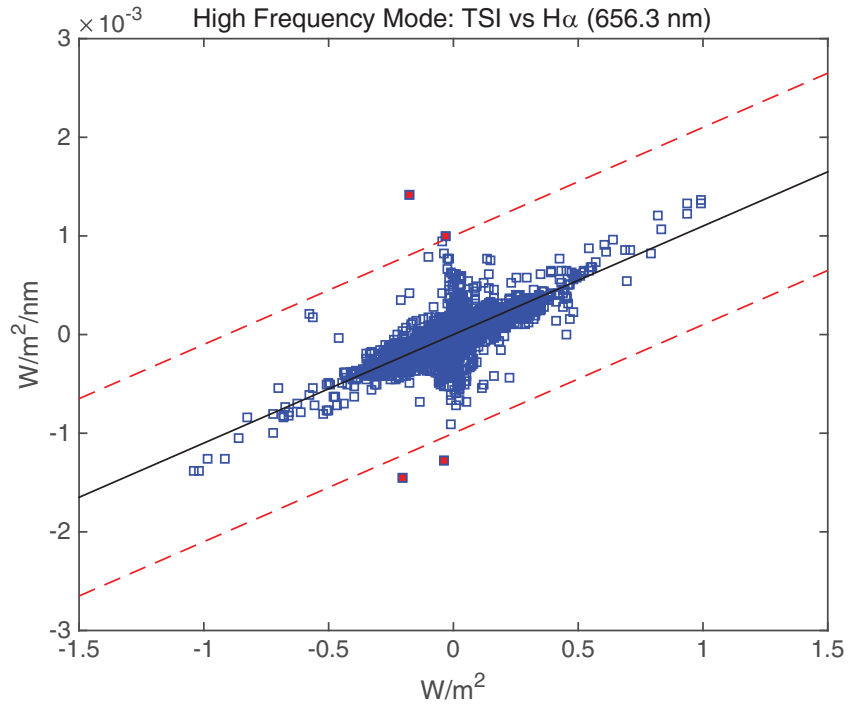

Fig. 6. Scatter plot of high frequency mode variation of TSI and SIM Hydrogen- $\alpha$ line at $656.3 \mathrm{~nm}$. The slope of the regression line is 0.0011 , which is visibly indistinguishable from the 1 to 1000 ratio line. The red lines represent the deviation of the high frequency variation of Hydrogen- $\alpha$ from its regression to TSI when the deviation is equal to $\pm 0.001 \mathrm{~W} \mathrm{~m}^{-2} \mathrm{~nm}^{-1}$. The red squares denote estimated outliers of SIM/SSI at $\mathrm{H}-\alpha$. These outliers are excluded from the SSI data for EEMD analysis. 
J.N. Lee et al.: Solar rotational modulations of spectral irradiance
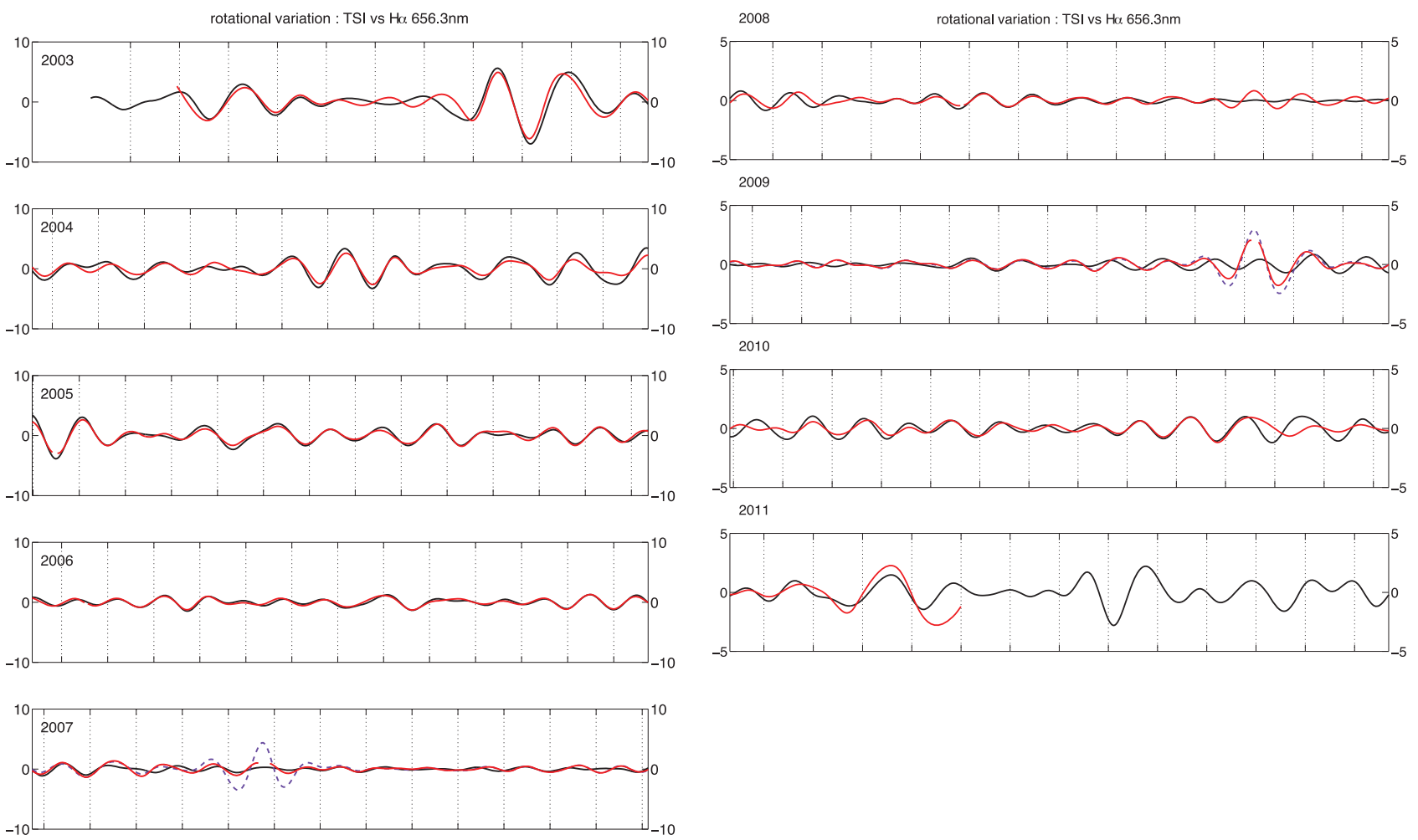

Fig. 7. Same as Figure 4, but comparison of rotational variation from the TIM TSI (black), SIM Hydrogen- $\alpha$ corrected (red), and SIM Hydrogen- $\alpha$ uncorrected (purple dots) at $656.3 \mathrm{~nm}$ band. For corrected SIM data, only a few outliers from SIM daily data as shown in Figure 6 are excluded before EEMD analysis.

rotational variation of UV and TSI during high solar activity period.

The close agreement of the rotational mode from SOLSTICE and SIM is encouraging, as low frequency solar cycle variability is quite different in those two (Harder et al. 2005, 2009). Recent studies suggest that incomplete accounting for instrument degradation and degradation correction may contribute to the off-phase of 11-year solar cycle trends in SIM measured SSI (Ball et al. 2011; Deland \& Cebula 2012; Lean \& DeLand 2012; Ermolli et al. 2013). The significant differences in SSI variability between SORCE and other observed and modeled SSI data (Ermolli et al. 2013) indicate that there is still uncertainty in our knowledge of how the solar irradiance varies in different wavelengths. The long-term instrument degradation truly exists in SSI measurements and may affect the high and low frequency variations of SSI, but this effect may be reduced in rotational variations by screening other modes with longer and shorter periods.

To identify and remove outliers from SSI in VIS, a scatter plot of high frequency mode variations of TSI and SIM $656.3 \mathrm{~nm}$ band near Hydrogen- $\alpha$ was created, as seen in Figure 6 . The slope of the regression line is 0.0011 , which is visibly indistinguishable from the 1 to 1000 ratio line. The red lines represent the deviation of the high frequency variation of Hydrogen- $\alpha$ from its regression to TSI when the deviation is equal to $\pm 0.001 \mathrm{~W} \mathrm{~m}^{-2} \mathrm{~nm}^{-1}$. The red squares denote estimated outliers of SIM/SSI at $\mathrm{H}-\alpha$. The red squares denote estimated outliers of SIM/SSI near H- $\alpha(656.3 \mathrm{~nm})$. The outliers in the SIM data during the data gaps in 2007 and 2009 are related to times when SIM did not have temperaturestabilized measurements due to the SORCE spacecraft being in its safe hold mode during satellite anomaly resolution. These outliers are excluded from the SSI data for EEMD analysis.

Similar to Figures 4 and 5, the comparison of rotational variations from the TIM TSI (black) and SIM Hydrogen- $\alpha$ line is shown in Figure 7. The rotational variations of the Hydrogen- $\alpha$ line at $656 \mathrm{~nm}$ track TSI variations with the same phase. After removing a few outliers in 2007 and 2009, the corrected SIM modes (in red) provide better agreement with those of TSI in 2007. The suspicious modes in uncorrected SIM in 2007 and 2009 are plotted as purple dots. For corrected SIM data, only a few outliers from SIM daily data as shown in Figure 6 are excluded before EEMD analysis is applied. Several outstanding rotational modes still remain in late 2009 , regardless of the correction.

The TSI variability can be decomposed with at least two components; dark sunspots and bright faculae (Lean et al. 2005). In order to quantify the spectral dependence of the rotational variations of SSI and its correlation to each of darkening and brightening effect, the linear correlation between the SSI modes and modes of sunspot area and Mg II index are calculated for each wavelength in 120-1600 nm. The sunspot area on the solar disk ${ }^{2}$ on a given day is used as a proxy for the darkening effect of sunspots. The $\mathrm{Mg}$ II index is used as a proxy for brightening effects of faculae/plages. The Mg II index is the ratio of measurements from the core of the $\mathrm{K}$ and $\mathrm{K} \mathrm{Mg}$ II emission lines at $280 \mathrm{~nm}$ to measurements in the nearby wings (278 and $282 \mathrm{~nm}$ ). The variability in the $\mathrm{Mg}$ II index is attributed to chromospheric extensions of the

\footnotetext{
${ }^{2}$ http://www.ngdc.noaa.gov/stp/spaceweather.html
} 
(a)

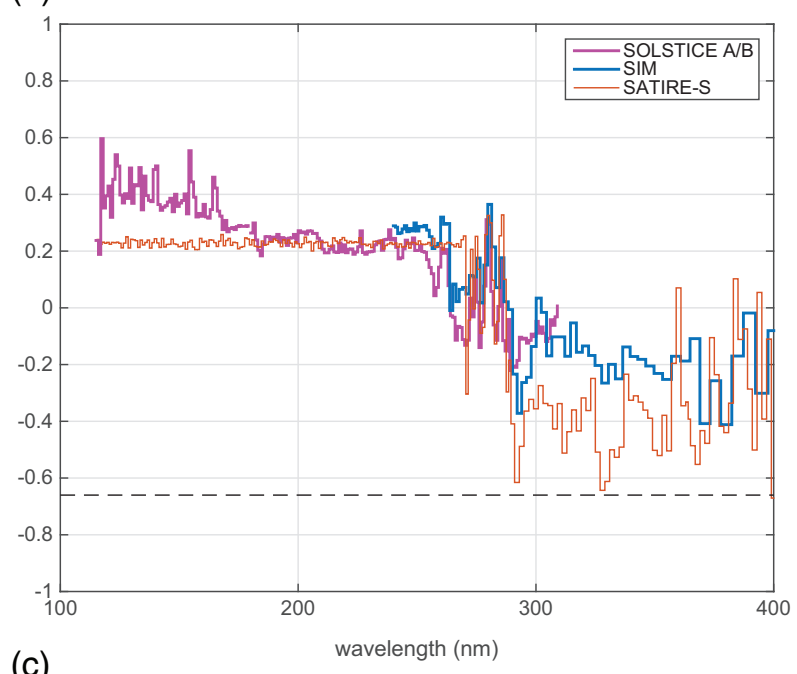

(c)

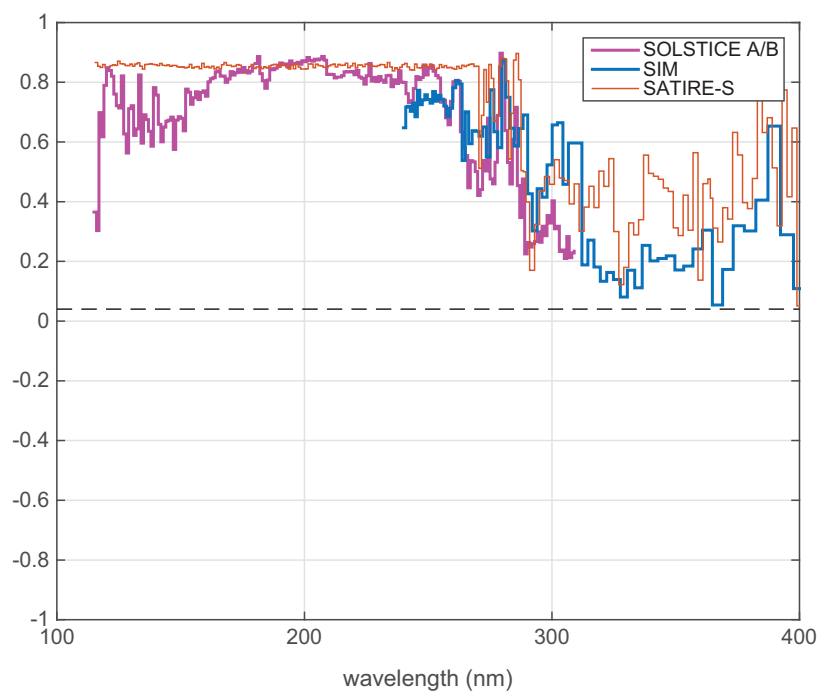

(b)

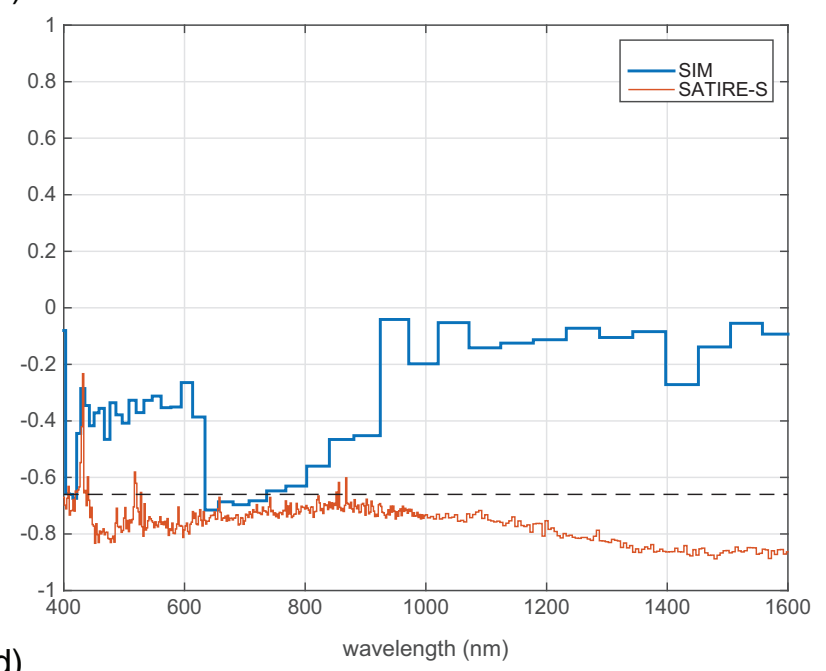

(d)

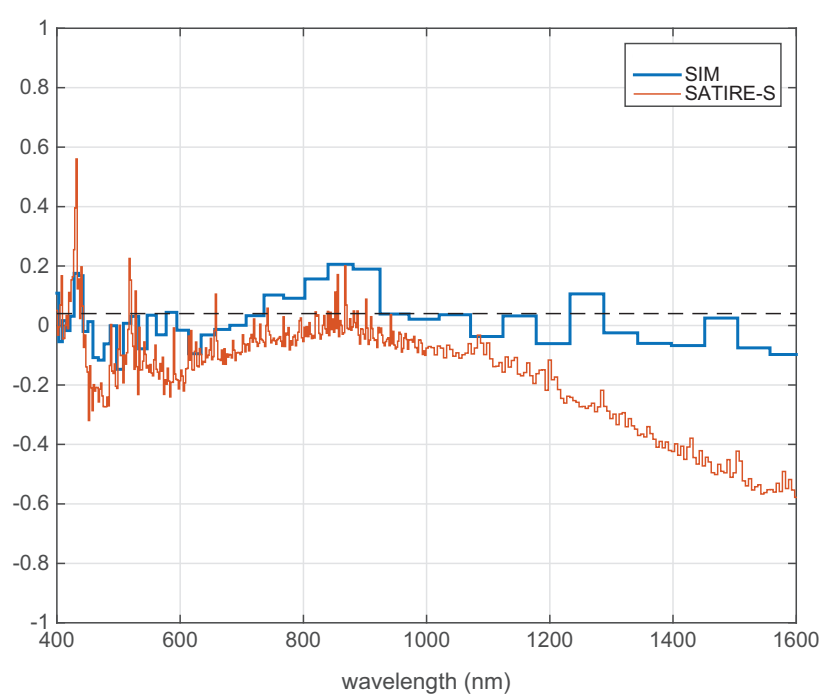

Fig. 8. Comparison of linear correlations of rotational variation between SSI and two proxies; sunspot area (Figs. $8 \mathrm{a}$ and $8 \mathrm{~b}$ ) and Mg II index (Figs. 8c and 8d). The blue lines denote the correlation between SIM SSI and the proxies, the red lines denote the correlations between SATIRE-S modeled SSI and the proxies, and the magenta lines denote the correlations between SOLSTICE A/B SSI and the proxies. Overlaid black dotted lines denote the correlations between TIM TSI and the proxies, respectively. (a) Correlation of the 27-day mode between SSI and sunspot area (115-400 nm). (b) Correlation of the 27-day mode between SSI and sunspot area (400-1600 nm). (c) Correlation of the 27-day mode between SSI and MgII index (115-400 nm). (d) Correlation of the 27-day mode between SSI and MgII index (400-1600 nm).

photospheric faculae (Viereck et al. 2001). For this study, we use the University of Bremen's Mg II composite. ${ }^{3}$

Figures $8 \mathrm{a}-8 \mathrm{~d}$ show the correlation plots of the rotational mode of SSI from OLSTICE, SIM, and SATIRE-S with respect to the modes of sunspot and Mg II index. The blue curve denotes the correlation between SIM SSI and solar proxies, and the red curve denotes the correlation between SATIRE modeled SSI and solar proxies. Overlaid magenta curve denotes the correlation between SOLSTICE A/B and solar proxies. The correlation with SIM is calculated for the entire SIM period (2003-2011) and the correlation with SATIRE SSI is calculated for 2003-August 2013. The correlations between the modes of proxies and TSI are also shown in black dotted lines. The rotational modes of sunspot area and TSI are negatively correlated $(\gamma=-0.66)$, while the modes of Mg II index and TSI are nearly zero $(\gamma=-0.04)$.

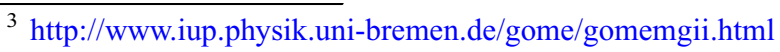

It is noticeable that there is a strong wavelength dependence in the SSI rotational variations in both cases. In particular, it is found that for regions of UV shorter than $300 \mathrm{~nm}$, the brightening effect dominates the rotational variation of SSI. The correlations between rotational variations of SSI and Mg II index are very high $(\gamma>0.8)$ in the UV region (Fig. 8c), while the correlations with sunspot area are not significant $(\gamma \sim 0.2)$ (Fig. 8a). These correlations imply that brightening effect accounts for more than $60 \%$ of the rotational variance in FUV and MUV below $300 \mathrm{~nm}$. Only 4\% of the rotational variance of UV can be attributed to the darkening effect. These low correlations between UV and TSI modes are already shown in Figure 3.

Different from the Mg II index correlations, the SSI mode correlations with sunspot area mode are low in the UV region, but increase with wavelength above $300 \mathrm{~nm}$ and reach high negative correlation of -0.6 for SIM and over -0.8 for SATIRE near $400 \mathrm{~nm}$ (Fig. 8b). While this high correlation 
is maintained throughout the spectrum in VIS and NIR for SATIRE, SIM correlation drops to a coefficient of -0.4 in $400-600 \mathrm{~nm}$, and rises again in the wavelength region 600-800 nm. Consistent high correlations for SATIRE indicate the clear shift from faculae dominated variability in the UV to sunspot dominated variability above approximately $400 \mathrm{~nm}$.

The high correlations of Mg II index in UV decrease with wavelength; the high correlations of $\mathrm{Mg}$ II index decrease to near zero in VIS and NIR region (Fig. 8d). These near zero correlations are continued throughout the spectrum for SIM, but they increase with wavelength for SATIRE up to -0.6 .

In general, the correlation coefficients suggest that the agreement in rotational variations between the SSI model and observations is best in MUV in 180-300 nm. The agreement between the SORCE observations and SATIRE model is variable with wavelength. Model uncertainties are expected, due mainly to the assumption of local thermodynamic equilibrium (LTE) and the use of opacity distribution functions (ODFs) (Kurucz 1992). While large disagreements between observed and modeled SSI can be expected at some strong emission lines i.e., $\mathrm{Mg}$ II at $280 \mathrm{~nm}$, and $\mathrm{Mg}$ I at $285 \mathrm{~nm}$, or the complex sets of lines around 240 and $250 \mathrm{~nm}$, SATIRE shows good agreement with SOLSTICE in 210-260 nm, and with SIM above $260 \mathrm{~nm}$. Low correlation of the modes from the SIM and SATIRE data in 420-600 nm and above $900 \mathrm{~nm}$ implies that there exists an apparent gap between the observation and model in VIS and NIR region.

In the NIR region $(1000 \mathrm{~nm}-1600 \mathrm{~nm})$, the negative correlations in SATIRE modes with sunspot area modes (Fig. 8b) are consistently high throughout the wavelength above $400 \mathrm{~nm}$, while the correlation in SIM is down to near zero above $900 \mathrm{~nm}$. The latter values can partly be attributed to the temperature-induced sensitivity problems for SIM in VIS region above $850 \mathrm{~nm}$ (Harder et al. 2005). In SATIRE$\mathrm{S}$, the faculae brightenings get weaker in VIS in rotational timescales, i.e., the faculae produce most of the brightening when near the limb, and very little or even no brightening when at disk center (Unruh et al. 2008). In the NIR, the facular brightenings become very weak and might even disappear completely for the faculae model in SATIRE-S. Combined with the sunspot contribution, these faculae brightening and weakening effects in the model can lead to the familiar sunspot dominated features similar to the TSI variations. High correlations of rotational variation between TSI and SATIRE-S indicate that rotational variation in TSI can be mainly explained by the evolution of photospheric magnetic fields, as suggested by Ball et al. (2011).

\section{Conclusions}

Solar rotational modulation of SSI is identified and its correlation with the variability of TSI is shown. During the last decade, the SORCE SOLSTICE and SIM spectral irradiance observations have precision sufficient to identify solar rotational modulation at wavelengths between 120 and $2400 \mathrm{~nm}$. The SORCE observations revealed that no portion of the spectrum in the Sun's radiative output is invariant with time. The EEMD analysis is applied to the TSI and SSI data acquired from TIM, SOLSTICE, SIM, SEE, and SATIRE-S to extract significant modes of the time-varying solar irradiance time series. This method is quite effective to extract internal modes of TSI and SSI variability, including the solar rotational signature that is periodic but may not be sinusoidal.
Coherent $\sim 30$-day variations in the solar irradiance time series are considered as the 27-day solar rotational cycle in this study. The appearance of the rotational variations is reliable enough that each cycle can be identified with current space-borne TSI and SSI observations.

Over all, the rotational variations of SSI measured by SOLSTICE and SIM instruments are in good agreement with a linear correlation of $\sim 0.65$ at $240 \mathrm{~nm}$ during their overlapping observational periods (2003-2011). This suggests the presence of rotational variations is coherent between the two independent UV observations and represents the solar rotational cycle with high confidence. The rotational variations of sunspot area and TSI are highly correlated with those of SSI in VIS spectral region, where wavelength is between 600 and $800 \mathrm{~nm}$. However, the rotational variations of UV show low correlation with those of sunspot area and TSI, but high correlation with those of $\mathrm{Mg}$ II index.

Highly correlated relationships between solar UV irradiance and solar proxy indices have already been shown (Lockwood et al. 2010; Marchenko \& DeLand 2014) but these correlations were not always consistent due to their dependence on timescales (Dudok de Wit et al. 2009). Thus, the phase relationships between SSI and TSI obtained from rotational variations cannot necessarily be applied to the relationships with 11-year or longer timescale variations.

For about 6 months after July 2013, SORCE was operating in "safe hold" mode due to declining battery levels, but in February 2014 transitioned to a "Day Only Operations" mode, operating in "safe hold" during the eclipse portion of each orbit, when the satellite is shaded from the Sun by Earth, but doing normal science data collection during the daylight portion of each orbit. As long as the solar panels work, SORCE will continue to extend its 13-year record of TSI and SSI observations. The Total and Spectral Solar Irradiance Sensor-1 (TSIS-1) mission plan is underway to extend the TSI and SSI observations. To fill any possible gap in the future solar irradiance record between SORCE and TSIS, the Total Solar Irradiance Calibration Transfer Experiment (TCTE) has been operating since December 2013 to provide a cross-calibration between the SORCE TIM and the new TSIS TIM, which is due for deployment on the International Space Station by 2018. ${ }^{4}$ A reliable assessment of solar forcing on the Earth's climate depends on continuous and absolutely calibrated total and spectral solar irradiance records. The value of the longterm solar irradiance data, both TSI and SSI, cannot be overemphasized, along with other fundamental Earth observing measurements that are monitoring Earth's changing climate.

Acknowledgements. This work is supported by the NASA living with a Star Targeted Research and Technology Program (NNH10ZDA001 N-LWSTRT). We wish to thank Natalie Krivova, Yvonne Unruh, and two reviewers for many useful comments which helped to improve the manuscript and our analysis. We also thank the SORCE, TIMED, and SATIRE teams for providing their data and analysis support. The editor thanks Tom Woods and an anonymous referee for their assistance in evaluating this paper.

\section{References}

Andrews, M.B., J.R. Knight, and L.J. Gray. A simulated lagged response of the North Atlantic Oscillation to the solar cycle over the period 1960-2009. Environ. Res. Lett., 10, 054022, 2015, DOI: $10.1088 / 1748-9326 / 10 / 5 / 054022$.

\footnotetext{
${ }^{4}$ http://science.nasa.gov/missions/tsis-1/
} 
Ball, W.T., Y.C. Unruh, N.A. Krivova, S. Solanki, and J.W. Harder. Solar irradiance variability: a six-year comparison between SORCE observations and the SATIRE model. Astron. Astrophys., 530, A71, 2011, DOI: 10.1051/0004-6361/201016189.

Ball, W.T., N.A. Krivova, Y.C. Unruh, J.D. Haigh, and S. Solanki. A new SATIRE-S spectral solar irradiance reconstruction for solar cycles 21-23 and its implications for stratospheric ozone. J. Atmos. Sci., 71, 4086-4101, 2014,

DOI: $10.1175 /$ JAS-D-13-0241.1.

Barnhart, B.L., and W.E. Eichinger. Analysis of sunspot variability using the Hilbert-Huang transform. Sol. Phys., 269, 439-449, 2011, DOI: 10.1007/s11207-010-9701-6.

Cook, J.W., G.E. Brueckner, and M.E. Vanhoosier. Variability of the solar flux in the ultraviolet 1175-2100 ̊. J. Geophys. Res., 85 2257-2268, 1980, DOI: 10.1029/JA085iA05p02257.

Deland, M.T., and R.P. Cebula. Solar UV variations during the decline of Cycle 23. J. Atmos. Sol. Terr. Phys., 77, 225-234, 2012, DOI: 10.1016j.jastp.2012.01.007.

Dikty, S., M. Weber, C. von Savigny, T. Sonkaew, A. Rozanov, and J.P. Burrows. Modulations of the 27 day solar rotation signal in stratospheric ozone from Scanning Imaging Absorption Spectrometer for Atmospheric Cartography (SCIAMACHY) (2003-2008). J. Geophys. Res., 115, 2010, DOI: 10.1029/2009JD012379.

Domingo, V., I. Ermolli, P. Fox, C. Fröhlich, M. Haberreiter, et al. Solar surface magnetism and irradiance on time scales from days to the 11-year cycle. Space Sci. Rev., 145, 337-380, 2009, DOI: $10.1007 / \mathrm{s} 11214-009-9562-1$.

Dudok de Wit, T., M. Kretzschmar, J. Lilensten, and T. Woods. Finding the best proxies for the solar UV irradiance. Geophys. Res. Lett., 36, L10107, 2009, DOI: 10.1029/2009GL037825.

Ermolli, I., K. Matthes, T. Dudok de Wit, N.A. Krivova, K. Tourpali, et al. Recent variability of the solar spectral irradiance and its impact on climate modeling. Atmos. Chem. Phys., 13, 3945-3977, 2013, DOI: 10.5194/acp-13-3945-2013.

Fioletov, V.E. Estimating the 27-day and 11-year solar cycle variations in tropical upper stratospheric ozone. J. Geophys. Res., 114, D02302, 2009, DOI: 10.1029/2008JD010499.

Floyd, L.E., J.W. Cook, L.C. Herring, and P.C. Crane. SUSIM'S 11-year observational record of the solar UV irradiance. $A d v$. Space Res., 31, 2111-2120, 2003, DOI: $10.1016 / \mathrm{S} 0273-1177(03) 00148-0$

Fontenla, J.M., J. Harder, W. Livingston, M. Snow, and T. Woods. High resolution solar spectral irradiance from extreme ultraviolet to far infrared. J. Geophys. Res., 116, D20108, 2011, DOI: 10.1029/2011JD016032.

Fröhlich, C., and J. Lean Solar radiative output and its variability: evidence and mechanisms. Astron. Astrophys. Rev., 12, 273-320, 2004, DOI: 10.1007/s00159-004-0024-1.

Gray, L.J., J. Beer, M. Geller, J.D. Haigh, and M. Lockwood. Solar influences on climate. Rev. Geophys., 48, RG4001, 2010, DOI: 10.1029/2009RG000282.

Haigh, J.D. The Sun and the Earth's Climate. Living Rev. Sol. Phys., 4, 2, 2007, DOI: 10.12942/lrsp-2007-2.

Haigh, J.D., A.R. Winning, R. Toumi, and J.W. Harder. An influence of solar spectral variations on radiative forcing of climate. Nature, 467, 696-699, 2010, DOI: 10.1038/nature09426.

Harder, J., G. Lawrence, J. Fontenla, G. Rottman, and T. Woods. The spectral irradiance monitor: scientific requirements, instrument design, and operation modes. Sol. Phys., 230, 141-167, 2005, DOI: $10.1007 / \mathrm{s} 11207-005-5007-5$.

Harder, J.W., J.M. Fontenla, P. Pilewskie, E.C. Richard, and T.N Woods. Trends in solar spectral irradiance variability in the visible and infrared. Geophys. Res. Lett., 36, L07801, 2009, DOI: 10.1029/2008GL036797.

Huang, N.E., Z. Shen, S.R. Long, M.C. Wu, H.H. Shih, Q. Zheng, N.-C. Yen, C.C. Tung, and H.H. Liu. The empirical mode decomposition and the Hilbert spectrum for nonlinear and nonstationary time series analysis. Proc. R. Soc., Ser. A, 454, 903-995, 1998
Ineson, S., A.C. Maycock, L.J. Gray, A.A. Scaife, N. Dunstone, et al. Regional climate impacts of a possible future grand solar minimum. Nat. Commun., 6, 7535, 2015, DOI: $10.1038 /$ ncomms 8535 .

Kodera, K. Solar cycle modulation of the North Atlantic Oscillation: implications in the spatial structure of the NAO. Geophys. Res. Lett., 29 (8), 1218, 2002, DOI: 10.1029/2001GL014557.

Kopp, G., and G. Lawrence. The Total Irradiance Monitor (TIM): instrument design. Sol. Phys., 230, 91-109, 2005, DOI: $10.1007 / \mathrm{s} 11207-005-7446-4$

Kopp, G., and J.L. Lean. A new, lower value of total solar irradiance: evidence and climate significance. Geophys. Res. Lett., 38, L01706, 2011, DOI: 10.1029/2010GL045777.

Kopp, G., K. Heuerman, D. Harber, and V. Drake. The TSI radiometer facility - absolute calibrations for total solar irradiance instruments. Proc. SPIE, 6677, 667709, 2007, DOI: $10.1117 / 12.734553$.

Kopp, G., A. Fehlmann, W. Finsterle, D. Harber, and K. Heuerman. Total solar irradiance data record accuracy and consistency improvements. Metrologia, 49, S29-S33, 2012, DOI: MET/407161/SPE/275237.

Kren, A.C., D.R. Marsh, A.K. Smith, and P. Pilewskie. Examining the stratospheric response to the solar cycle in a coupled WACCM simulation with an internally generated QBO. Atmos. Chem. Phys., 14, 4843-4856, 2014.

Krivova, N.A., and S.K. Solanki. Models of solar irradiance variations: current status. J. Astrophys. Astron., 29, 151-158, 2008.

Krivova, N.A., S.K. Solanki, and L. Floyd. Reconstruction of solar UV irradiance in cycle 23. Astron. Astrophys., 452, 631-639, 2006, DOI: 10.1051/0004-6361:20064809.

Krivova, N.A., S.K. Solanki, and Y.C. Unruh. Towards a long-term record of solar total and spectral irradiance. J. Atmos. Sol. Terr. Phys., 73, 223-234, 2011, DOI: 10.1016/j.jastp.2009.11.013.

Kurucz, R.L. Finding the missing solar ultraviolet opacity. Rev. Mex. Astron. Astrofis., 23, 181, 1992.

Lagg, A., S.K. Solanki, T.L. Riethmüller, V. Martínez Pillet, M. Schüssler, et al. Fully resolved quiet-sun magnetic flux tube observed with the SUNRISE/IMAX instrument. Astrophys. J. Lett., 723, L164, 2010.

Lawrence, G.M., G. Rottman, J. Harder, and T. Woods. Solar Total Irradiance Monitor: TIM. Metrologia, 37, 407-410, 2000.

Lean, J. Variations in the sun's radiative output. Rev. Geophys., 29, 505-535, 1991, DOI: 10.1029/91RG01895.

Lean, J. The Sun's variable radiation and its relevance for Earth. Annu. Rev. Astron. Astrophys., 35, 33-67, 1997, DOI: 10.1146 /annurev.astro.35.1.33.

Lean, J., and M.T. DeLand. How does the Sun's spectrum vary? J. Climate, 25, 2555-2560, 2012 DOI: $10.1175 / J C L I-D-11-00571.1$

Lean, J., G. Rottman, J. Harder, and G. Kopp. SORCE contributions to new understanding of global change and solar variability. Sol. Phys., 230, 27-53, 2005, DOI: 10.1007/s11207-005-1527-2.

Lee, J.N., and S. Hameed. The Northern Hemisphere annular mode in summer: its physical significance and its relation to solar activity variations. J. Geophys. Res., 112, D15111, 2007, DOI: $10.1029 / 2007$ JD008394.

Lee, J.N., S. Hameed, and D.T. Shindell. Northern annular mode in summer and its relation to solar activity variations in the GISS Model E. J. Atmos. Sol. Terr. Phys., 70, 730-741, 2008, DOI: $10.1016 / j$.jastp.2007.10.012.

Lee, J.N., R.F. Cahalan, and D.L. Wu. The 27-Day rotational variations in total solar irradiance observations: from SORCE/ TIM, ACRIM III, and SOHO/VIRGO. J. Atmos. Sol. Terr. Phys., 132, 64-73, 2015, DOI: 10.1016/j.jastp.2015.07.001.

Li, K.-F., and K.-K. Tung. Quasi-Biennial oscillation and solar cycle influences on winter arctic total ozone. J. Geophys. Res., 119 , 5823-5835, 2014, DOI: 10.1002/2013JD021065.

Liang, H., Q.-H. Lin, and J.D.Z. Chen. Application of the empirical mode decomposition to the analysis of esophageal manometric 
data in gastroesophageal reflux disease. IEEE Trans. Biomed. Eng., 52, 10, 2005.

Lockwood, M., C. Bell, T. Woollings, R.G. Harrison, L.J. Gray, and J.D. Haigh. Top-down solar modulation of climate: evidence for centennial-scale change. Environ. Res. Lett., 5, 034008, 2010, DOI: $10.1088 / 1748-9326 / 5$.

Marchenko, S., and M.T. DeLand. Solar spectral irradiance changes during cycle 24. Astrophys. J., 789, 117, 2014, DOI: $10.1088 / 0004-637 X / 789 / 2 / 117$

Merkel, A.W., J.W. Harder, D.R. Marsh, A.K. Smith, J.M. Fontenla, and T.N. Woods. The impact of solar spectral irradiance variability on middle atmospheric ozone. Geophys. Res. Lett., 38, L13802, 2011, DOI: 10.1029/2011GL047561.

McClintock, W.E., G. Rottman, and T.N. Woods. Solar-stellar irradiance comparison experiment II (SOLSTICE II): instrument concept and design. Sol. Phys., 230, 225, 2005a, DOI: $10.1007 / 0-387-37625-9 \_12$.

McClintock, W.E., M. Snow, and T.N. Woods. Solar-stellar irradiance comparison experiment II (SOLSTICE II): pre-launch and on-orbit calibrations. Sol. Phys., 230, 259, 2005b, DOI: $10.1007 / \mathrm{s} 11207-005-1585-5$.

Preminger, D.G., and S.R. Walton. A new model of total solar irradiance based on sunspot areas. Geophys. Res. Lett., 32 , L14109, 2005, DOI: 10.1029/2005GL022839.

Rempel, M., and R. Schlichenmaier. Sunspot modeling: from simplified models to radiative MHD simulations. Living Rev. Sol. Phys., 8, 3, 2011, DOI: 10.12942/lrsp-2011-3.

Rottman, G. Solar ultraviolet irradiance and its temporal variations. J. Atmos. Sol. Terr. Phys., 61, 37-44, 1999, DOI: 10.1016/S1364-6826(98)00014-X.

Rottman, G. Measurements of total and spectral solar irradiance. Space Sci. Rev., 125, 39, 2006, DOI: 10.1007/s11214-006-9045-6.

Rottman, G., T.W. Woods, M. Snow, and G. DeToma. The solar cycle variation in ultraviolet irradiance. Adv. Space Res., 27, 1927-1932, 2001, DOI: 10.1016/S0273-1177(01)00272-1.

Rottman, G., L. Floyd, and R. Viereck. Measurement of the solar ultraviolet irradiance in solar variability and its effect on climate. Geophysical Monograph, 141, 111-125, 2004.

Rottman, G., T.W. Woods, and V. George. Solar radiation and climate experiment. Sol. Phys., 230, 185, 2005.

Ruzmaikin, A., M.L. Santee, M.J. Schwartz, L. Froidevaux, and H.M. Pickett. The 27-day variations in stratospheric ozone and temperature: new MLS data. Geophys. Res. Lett., 34, L02819, 2007, DOI: 10.1029/2006GL028419.

Shapiro, A.I., S.K. Solanki, N.A. Krivova, R.V. Tagirov, and W.K. Schmutz. The role of the Fraunhofer lines in solar brightness variability. Astron. Astrophys., 581, A116, 2015.

Shindell, D.T., G.A. Schmidt, R.L. Miller, and D. Rind. Northern Hemisphere winter climate response to greenhouse gas, ozone, solar, and volcanic forcing. J. Geophys. Res., 106, 7193-7210, 2001, DOI: 10.1029/2000JD900547.

Snow, M., W.E. McClintock, G. Rottman, and T.N. Woods. Solarstellar irradiance comparison experiment II (SOLSTICE II): examination of the solar stellar comparison technique. Sol. Phys., 230, 295, 2005.

Solanki, S.K. Sunspots: an overview. Astron. Astrophys. Rev., 11, 153-286, 2003.
Solanki, S.K., N.A. Krivova, and T. Wenzler. Irradiance models. Adv. Space Res., 35, 376-383, 2005.

Spruit, H.C. The solar engine and its influence on terrestrial atmosphere and climate. NATO ASI Series I, vol. 25, Kluwer, Dordrecht, 107, 1994.

Stephens, G.L., J.L. Li, M. Wild, C.A. Clayson, N. Loeb, S. Kato, T. L'Ecuyer, P.W. Stackhouse, and T. Andrews. The energy balance of the earth's climate system. Nat. Geosci., 5, 691-696, 2012, DOI: $10.1038 /$ ngeo 1580.

Swartz, W.H., R.S. Stolarski, L.D. Oman, E.L. Fleming, and C.H. Jackman. Middle atmosphere response to different descriptions of the 11-yr solar cycle in spectral irradiance in a chemistryclimate model. Atmos. Chem. Phys., 12, 5937-5948, 2012, DOI: $10.5194 /$ acp-12-5937-2012.

Topka, K.P., T.D. Tarbell, and A.M. Title. Properties of the smallest solar magnetic elements. II. Observations versus hot wall models of faculae. Astrophys. J., 484, 479, 1997.

Unruh, Y.C., S.K. Solanki, and M. Fligge. The spectral dependence of facular contrast and solar irradiance variations. Astron. Astrophys., 345, 635-642, 1999.

Unruh, Y.C., N. Krivova, S. Solanki, J. Harder, and G. Kopp. Spectral irradiance variations: comparisons between observations and the SATIRE model on solar rotation time scales. Astron. Astrophys., 486, 311, 2008, DOI: $10.1051 / 0004-6361: 20078421$

Viereck, R., L. Puga, D. McMullin, D. Judge, M. Weber, and W.K. Tobiska. A proxy for solar EUV. Geophys. Res. Lett., 28 , 1343-1346, 2001.

Wehrli, C., W. Schmutz, and A.I. Shapiro. Correlation of spectral solar irradiance with solar activity as measured by VIRGO. Astron. Astrophys., 556, L3, 2013, DOI: $10.1051 / 0004-6361 / 201220864$.

Wen, G., R.F. Cahalan, J.D. Haigh, P. Pilewskie, L. Oreopoulos, and J.W. Harder. Reconciliation of modeled climate responses to spectral solar forcing. J. Geophys. Res. [Atmos.], 118, 6281-6289, 2013, DOI: 10.1002/jgrd.50506.

Wild, M., D. Folini, C. Schär, N. Loeb, E. Dutton, and G. KönigLanglo. The global energy balance from a surface perspective. Clim. Dyn., 323, 3107, 2012, DOI: $10.1007 / \mathrm{s} 00382-012-1569-8$

Woods, T.N., F.G. Eparvier, S.M. Bailey, P.C. Chamberlin, J. Lean, G.J. Rottman, S.C. Solomon, W.K. Tobiska, and D.L. Woodraska. Solar EUV experiment (SEE): mission overview and first results. J. Geophys. Res., 110, A01312, 2005, DOI: $10.1029 / 2004 J A 010765$

Woods, T.N., M. Snow, J. Harder, G. Chapman, and A. Cookson. A different view of solar spectral irradiance variations: modeling total energy of six-month intervals. Sol. Phys., 290, 2649-2676, 2015, DOI: 10.1007/s11207-015-0766-0.

Wu, Z., and N.E. Huang. A study of the characteristics of white noise using the empirical mode decomposition method. Proc. $R$. Soc., Ser. A, 460, 1597-1611, 2004.

Yeo, K.L., N.A. Krivova, S.K. Solanki, and K.H. Glassmeier. Reconstruction of total and spectral solar irradiance since 1974 based on KPVT, SoHO/MDI and SDO/HMI observations. Astron. Astrophys., 570, A85, 2014, DOI: $10.1051 / 0004-6361 / 201423628$.

Cite this article as: Lee JN, Cahalan RF \& Wu DL. Solar rotational modulations of spectral irradiance and correlations with the variability of total solar irradiance. J. Space Weather Space Clim., 6, A33, 2016, DOI: 10.1051/swsc/2016028. 